\title{
On the curvature ellipse of minimal surfaces in $N^{3}(c) \times R$
}

\author{
Makoto Sakaki
}

\begin{abstract}
We discuss the curvature ellipse of minimal surfaces in the product space $N^{3}(c) \times R$, where $N^{3}(c)$ is the 3-dimensional simply connected space form of constant curvature $c$.
\end{abstract}

\section{Introduction}

Let $N^{n}(c)$ denote the $n$-dimensional simply connected space form of constant curvature $c$. When $c>0, N^{n}(c)$ is the $n$-dimensional sphere $S^{n}(c)$ of constant curvature $c$. When $c<0, N^{n}(c)$ is the $n$-dimensional hyperbolic space $H^{n}(c)$ of constant curvature $c$. When $c=0, N^{n}(c)$ is the $n$-dimensional Euclidean space $R^{n}$.

For a surface $M$ in a Riemannian manifold, the curvature ellipse at $p \in M$ is defined as

$$
E(p)=\left\{h(X, X)\left|X \in T_{p} M,\right| X \mid=1\right\},
$$

where $h$ is the second fundamental form of $M$. The notion of the curvature ellipse plays an important role in the geometry of surfaces in $N^{n}$ (c) (cf. [9], [14]). In particular, when the curvature ellipse is a circle at any point, the surface is called isotropic or superconformal (cf. [2], [5]). On the other hand, surfaces and submanifolds in the product space $N^{n}(c) \times R$ have been studied actively (cf. [1], [3], [4], [6], [7], [8], [12], [13]).

Received by the editors in June 2014.

Communicated by F. Bourgeois.

2010 Mathematics Subject Classification : 53A10, 53B25, 53C42.

Key words and phrases : minimal surface, product space, curvature ellipse. 
In this paper we discuss the curvature ellipse of minimal surfaces in $N^{3}(c) \times R$. The results are stated as follows:

Theorem 1.1. Let $M$ be a minimal surface in $N^{3}(c) \times R$ where $c \neq 0$. If the curvature ellipse is a circle at any point, then $M$ is totally geodesic.

Remark. When $c=0$, for a minimal surface in $R^{4}$, the curvature ellipse is a circle at any point if and only if the surface is a complex curve in $R^{4}$ with respect to some orthogonal complex structure. It can be seen, for example, by combining $[11$, Th.A] and [10, Th.5.3 or 5.4].

Theorem 1.2. There exists no minimal surface in $N^{3}(c) \times R$ with $c \neq 0$ such that the semi-major axis and the semi-minor axis of the curvature ellipse are both positive constant.

Remark. There are minimal surfaces in $S^{3}(c) \times R$ such that the semi-major axis of the curvature ellipse is positive constant and the semi-minor axis of the curvature ellipse is zero, which are minimal constant angle surfaces (cf. [3]).

\section{Preliminaries}

Let $N^{3}(c)$ be the 3-dimensional simply connected space form of constant curvature $c$. The curvature tensor $\bar{R}$ of $N^{3}(c) \times R$ satisfies

$$
\begin{aligned}
& \langle\bar{R}(X, Y) Z, W\rangle= \\
& \quad c\{\langle d \pi(Y), d \pi(Z)\rangle\langle d \pi(X), d \pi(W)\rangle-\langle d \pi(X), d \pi(Z)\rangle\langle d \pi(Y), d \pi(W)\rangle\},
\end{aligned}
$$

where $\pi: N^{3}(c) \times R \rightarrow N^{3}(c)$ is the projection map. Let $\xi$ denote the unit vector along $R$. Then we can see that

$$
\begin{aligned}
\bar{R}(X, Y) Z=c\{\langle Y, Z\rangle X-\langle X, Z\rangle Y-\langle Y, \xi\rangle\langle Z, \xi\rangle X+\langle X, \xi\rangle\langle Z, \xi\rangle Y \\
+\langle X, Z\rangle\langle Y, \xi\rangle \xi-\langle Y, Z\rangle\langle X, \xi\rangle \xi\} .
\end{aligned}
$$

We recall the method of moving frames for surfaces in $N^{3}(c) \times R$. Unless otherwise stated, we use the following convention on the ranges of indices:

$$
1 \leq A, B, \cdots \leq 4, \quad 1 \leq i, j, \cdots \leq 2, \quad 3 \leq \alpha, \beta, \cdots \leq 4
$$

Let $\left\{e_{A}\right\}$ be a local orthonormal frame field in $N^{3}(c) \times R$, and $\left\{\omega^{A}\right\}$ the dual coframe field. The connection forms satisfy

$$
d e_{B}=\sum_{A} \omega_{B}^{A} e_{A}
$$

Then $\omega_{B}^{A}+\omega_{A}^{B}=0$. The structure equations are given by

$$
d \omega^{A}=-\sum_{B} \omega_{B}^{A} \wedge \omega^{B},
$$




$$
d \omega_{B}^{A}=-\sum_{C} \omega_{C}^{A} \wedge \omega_{B}^{C}+\frac{1}{2} \sum_{C, D} \bar{R}_{A B C D} \omega^{C} \wedge \omega^{D},
$$

where

$$
\bar{R}_{A B C D}=\left\langle\bar{R}\left(e_{C}, e_{D}\right) e_{B}, e_{A}\right\rangle .
$$

Let $M$ be a surface in $N^{3}(c) \times R$. We choose the frame $\left\{e_{A}\right\}$ so that $\left\{e_{i}\right\}$ are tangent to $M$. Then $\omega^{\alpha}=0$ along $M$. In the following our argument will be restricted to $M$. By (2.3),

$$
0=-\sum_{i} \omega_{i}^{\alpha} \wedge \omega^{i}
$$

So there is a symmetric tensor $\left\{h_{i j}^{\alpha}\right\}$ such that

$$
\omega_{i}^{\alpha}=\sum_{j} h_{i j}^{\alpha} \omega^{j}
$$

where $h_{i j}^{\alpha}$ are the components of the second fundamental form of $M$.

We decompose the unit vector $\xi$ along $R$ as

$$
\xi=T+\eta
$$

where $T$ is tangent to $M$ and $\eta$ is normal to $M$. We say that $M$ is a constant angle surface (or a helix surface), if the tangent planes of $M$ make a constant angle with $\xi$, which is equivalent to that the length $|T|$ of $T$ is constant (cf. [3], [7], [12]). If $T=0$, then $M$ is a surface in a slice $N^{3}(c) \times\{*\}$. If $\eta=0$, then $M$ is a part of a cylinder, that is, a product of a regular curve in $N^{3}(c)$ and the factor $R$.

The Gaussian curvature $K$ and the normal curvature $K_{v}$ are given by

$$
d \omega_{2}^{1}=K \omega^{1} \wedge \omega^{2}, \quad d \omega_{4}^{3}=K_{v} \omega^{1} \wedge \omega^{2} .
$$

By (2.1), (2.4), (2.5), (2.6) and (2.7), we get

$$
K=c\left(1-|T|^{2}\right)+h_{11}^{3} h_{22}^{3}-\left(h_{12}^{3}\right)^{2}+h_{11}^{4} h_{22}^{4}-\left(h_{12}^{4}\right)^{2},
$$

and

$$
K_{v}=h_{11}^{3} h_{12}^{4}-h_{12}^{3} h_{11}^{4}+h_{12}^{3} h_{22}^{4}-h_{22}^{3} h_{12}^{4} .
$$

The mean curvature vector of $M$ is defined by

$$
H=\frac{1}{2} \sum_{\alpha}\left(h_{11}^{\alpha}+h_{22}^{\alpha}\right) e_{\alpha}
$$

We say that $M$ is minimal if $H=0$ on $M$.

We assume that $M$ is minimal. Then by (2.8) and (2.9),

$$
K=c\left(1-|T|^{2}\right)-\left(h_{11}^{3}\right)^{2}-\left(h_{12}^{3}\right)^{2}-\left(h_{11}^{4}\right)^{2}-\left(h_{12}^{4}\right)^{2},
$$

and

$$
K_{v}=2\left(h_{11}^{3} h_{12}^{4}-h_{12}^{3} h_{11}^{4}\right)
$$




\section{On the structure equations}

Let $M$ be a minimal surface in $N^{3}(c) \times R$. We use the notations in Section 2 . Suppose that either the curvature ellipse is not a circle at any point, or the curvature ellipse is a circle of positive radius at any point. Then we can choose the frame field $\left\{e_{A}\right\}$ so that

$$
\left(h_{i j}^{3}\right)=\left(\begin{array}{cc}
a & 0 \\
0 & -a
\end{array}\right), \quad\left(h_{i j}^{4}\right)=\left(\begin{array}{ll}
0 & b \\
b & 0
\end{array}\right),
$$

for some functions $a$ and $b$ with $|a| \geq|b|$. The semi-major axis of the curvature ellipse is $|a|$, and the semi-minor axis of the curvature ellipse is $|b|$. So, we have $|a|>|b|$ when the curvature ellipse is not a circle, and $|a|=|b|>0$ when the curvature ellipse is a circle of positive radius. Then by (2.6), (2.10) and (2.11),

$$
\begin{gathered}
\omega_{1}^{3}=a \omega^{1}, \quad \omega_{2}^{3}=-a \omega^{2}, \quad \omega_{1}^{4}=b \omega^{2}, \quad \omega_{2}^{4}=b \omega^{1}, \\
K=c\left(1-|T|^{2}\right)-a^{2}-b^{2}, \quad K_{v}=2 a b .
\end{gathered}
$$

Using (2.3), (2.4) and (3.1), we have

$$
\begin{gathered}
d \omega_{1}^{3}=d a \wedge \omega^{1}-a \omega_{2}^{1} \wedge \omega^{2} \\
=-\omega_{2}^{3} \wedge \omega_{1}^{2}-\omega_{4}^{3} \wedge \omega_{1}^{4}+\bar{R}_{3112} \omega^{1} \wedge \omega^{2} \\
=a \omega^{2} \wedge \omega_{1}^{2}-b \omega_{4}^{3} \wedge \omega^{2}+\bar{R}_{3112} \omega^{1} \wedge \omega^{2} .
\end{gathered}
$$

We can write

$$
T=T^{1} e_{1}+T^{2} e_{2}, \quad \eta=\eta^{3} e_{3}+\eta^{4} e_{4} .
$$

Then by (2.5) and (2.1), $\bar{R}_{3112}=c T^{2} \eta^{3}$. So, using the notation like

$$
\begin{array}{cl}
d a=a_{1} \omega^{1}+a_{2} \omega^{2}, & d b=b_{1} \omega^{1}+b_{2} \omega^{2}, \\
\omega_{2}^{1}=\left(\omega_{2}^{1}\right)_{1} \omega^{1}+\left(\omega_{2}^{1}\right)_{2} \omega^{2}, & \omega_{4}^{3}=\left(\omega_{4}^{3}\right)_{1} \omega^{1}+\left(\omega_{4}^{3}\right)_{2} \omega^{2},
\end{array}
$$

we get

$$
-a_{2}-2 a\left(\omega_{2}^{1}\right)_{1}+b\left(\omega_{4}^{3}\right)_{1}=c T^{2} \eta^{3} .
$$

Similarly, from the exterior derivatives of $\omega_{2}^{3}, \omega_{1}^{4}$ and $\omega_{2}^{4}$,

$$
\begin{gathered}
a_{1}-2 a\left(\omega_{2}^{1}\right)_{2}+b\left(\omega_{4}^{3}\right)_{2}=c T^{1} \eta^{3}, \\
b_{1}-2 b\left(\omega_{2}^{1}\right)_{2}+a\left(\omega_{4}^{3}\right)_{2}=c T^{2} \eta^{4}, \\
-b_{2}-2 b\left(\omega_{2}^{1}\right)_{1}+a\left(\omega_{4}^{3}\right)_{1}=-c T^{1} \eta^{4} .
\end{gathered}
$$

Let $\bar{\nabla}$ denote the Levi-Civita connection on $N^{3}(c) \times R$. Then, using (2.2), we have

$$
\begin{aligned}
& 0=\bar{\nabla} \xi=\bar{\nabla}\left(\sum_{i} T^{i} e_{i}+\sum_{\alpha} \eta^{\alpha} e_{\alpha}\right) \\
& =\sum_{i}\left(d T^{i}+\sum_{j} T^{j} \omega_{j}^{i}-\sum_{\alpha} \eta^{\alpha} \omega_{i}^{\alpha}\right) e_{i}
\end{aligned}
$$




$$
+\sum_{\alpha}\left(d \eta^{\alpha}+\sum_{i} T^{i} \omega_{i}^{\alpha}+\sum_{\beta} \eta^{\beta} \omega_{\beta}^{\alpha}\right) e_{\alpha} .
$$

So, using the notation like

$$
d T^{i}=\sum_{j} T_{j}^{i} \omega^{j}, \quad d \eta^{\alpha}=\sum_{j} \eta_{j}^{\alpha} \omega^{j}
$$

and (3.1), we have

$$
\begin{array}{cc}
T_{1}^{1}=-T^{2}\left(\omega_{2}^{1}\right)_{1}+a \eta^{3}, & T_{2}^{1}=-T^{2}\left(\omega_{2}^{1}\right)_{2}+b \eta^{4}, \\
T_{1}^{2}=T^{1}\left(\omega_{2}^{1}\right)_{1}+b \eta^{4}, & T_{2}^{2}=T^{1}\left(\omega_{2}^{1}\right)_{2}-a \eta^{3}, \\
\eta_{1}^{3}=-\eta^{4}\left(\omega_{4}^{3}\right)_{1}-a T^{1}, & \eta_{2}^{3}=-\eta^{4}\left(\omega_{4}^{3}\right)_{2}+a T^{2}, \\
\eta_{1}^{4}=\eta^{3}\left(\omega_{4}^{3}\right)_{1}-b T^{2}, \quad \eta_{2}^{4}=\eta^{3}\left(\omega_{4}^{3}\right)_{2}-b T^{1} .
\end{array}
$$

\section{Proof of Theorems}

In this section, using the notations in Sections 2 and 3, we prove Theorems 1.1 and 1.2 .

Proof of Theorem 1.1. Let $M$ be a minimal surface in $N^{3}(c) \times R$ where $c \neq 0$, and assume that the curvature ellipse at any point is a circle.

Suppose that $M$ is not totally geodesic. Then there exists a point $p \in M$ such that the curvature ellipse at $p$ is a circle of positive radius. So the curvature ellipse is a circle of positive radius on a neighborhood $U$ of $p$. We can use the argument in Section 3 on $U$, and $|a|=|b|>0$ on $U$. By the continuity, either $b=a \neq 0$ on $U$, or $b=-a \neq 0$ on $U$. In the case where $b=a \neq 0$ on $U$, by (3.3)-(3.6) and that $c \neq 0$, we have

$$
T^{1} \eta^{3}-T^{2} \eta^{4}=0, \quad T^{2} \eta^{3}+T^{1} \eta^{4}=0
$$

on $U$. Hence, noting also that $|T|^{2}+|\eta|^{2}=1$, we have either $T=0$ on $U$ or $\eta=0$ on $U$. In either case, since $|T|^{2}+|\eta|^{2}=1$ and $a \neq 0$ on $U$, we have a contradiction from (3.7)-(3.10). In the case where $b=-a \neq 0$ on $U$, similarly, we have a contradiction.

Therefore, $M$ has to be totally geodesic.

Proof of Theorem 1.2. Let $M$ be a minimal surface in $N^{3}(c) \times R$ where $c \neq 0$. Suppose that the semi-major axis and the semi-minor axis of the curvature ellipse are both positive constant. By Theorem 1.1, the curvature ellipse cannot be a circle of positive constant radius. So the curvature ellipse is not a circle at any point, and we can use the argument in Section 3, so that $a$ and $b$ are constant with $|a|>|b|>0$. Then (3.3)-(3.6) become

$$
\begin{aligned}
& -2 a\left(\omega_{2}^{1}\right)_{1}+b\left(\omega_{4}^{3}\right)_{1}=c T^{2} \eta^{3}, \\
& -2 a\left(\omega_{2}^{1}\right)_{2}+b\left(\omega_{4}^{3}\right)_{2}=c T^{1} \eta^{3},
\end{aligned}
$$




$$
\begin{aligned}
& -2 b\left(\omega_{2}^{1}\right)_{2}+a\left(\omega_{4}^{3}\right)_{2}=c T^{2} \eta^{4}, \\
& -2 b\left(\omega_{2}^{1}\right)_{1}+a\left(\omega_{4}^{3}\right)_{1}=-c T^{1} \eta^{4} .
\end{aligned}
$$

By (4.1)-(4.4) we obtain

$$
\begin{gathered}
-2 a \omega_{2}^{1}+b \omega_{4}^{3}=c \eta^{3}\left(T^{2} \omega^{1}+T^{1} \omega^{2}\right), \\
-2 b \omega_{2}^{1}+a \omega_{4}^{3}=c \eta^{4}\left(-T^{1} \omega^{1}+T^{2} \omega^{2}\right),
\end{gathered}
$$

and

$$
\begin{aligned}
2\left(\omega_{2}^{1}\right)_{1} & =-\frac{c}{a^{2}-b^{2}}\left(a T^{2} \eta^{3}+b T^{1} \eta^{4}\right), \\
2\left(\omega_{2}^{1}\right)_{2} & =\frac{c}{a^{2}-b^{2}}\left(b T^{2} \eta^{4}-a T^{1} \eta^{3}\right), \\
\left(\omega_{4}^{3}\right)_{1} & =-\frac{c}{a^{2}-b^{2}}\left(b T^{2} \eta^{3}+a T^{1} \eta^{4}\right), \\
\left(\omega_{4}^{3}\right)_{2} & =\frac{c}{a^{2}-b^{2}}\left(a T^{2} \eta^{4}-b T^{1} \eta^{3}\right) .
\end{aligned}
$$

Taking the exterior derivative of (4.5), and using (2.3), (2.7), (3.7)-(3.10), (4.7)-(4.10), we can get

$$
-2 a K+b K_{v}=c a\left(\frac{c}{a^{2}-b^{2}}|T|^{2}|\eta|^{2}+2\left(\eta^{3}\right)^{2}-|T|^{2}\right) .
$$

By (4.11), (3.2) and that $a \neq 0$,

$$
-2 c\left(1-|T|^{2}\right)+2 a^{2}+4 b^{2}=\frac{c^{2}}{a^{2}-b^{2}}|T|^{2}|\eta|^{2}+c\left\{2\left(\eta^{3}\right)^{2}-|T|^{2}\right\} .
$$

Similarly, by the exterior derivative of (4.6), we get

$$
-2 b K+a K_{v}=c b\left(2\left(\eta^{4}\right)^{2}-|T|^{2}-\frac{c}{a^{2}-b^{2}}|T|^{2}|\eta|^{2}\right) .
$$

By (4.13), (3.2) and noting that $b \neq 0$, we have

$$
-2 c\left(1-|T|^{2}\right)+4 a^{2}+2 b^{2}=c\left\{2\left(\eta^{4}\right)^{2}-|T|^{2}\right\}-\frac{c^{2}}{a^{2}-b^{2}}|T|^{2}|\eta|^{2} .
$$

By $(4.12)+(4.14)$,

$$
8 c|T|^{2}=6 c-6\left(a^{2}+b^{2}\right) .
$$

So $|T|$ is constant, and $M$ is a constant angle surface. Since $|a|>|b|>0$, $0<|T|<1$.

When $c<0$, by [12, Th. 3.2], $M$ is totally geodesic, which is a contradiction to $|a|>|b|>0$. When $c>0$, by [3, Lemma 2], we have $b=0$, which is also a contradiction to $|a|>|b|>0$. Thus we have proved Theorem 1.2.

Remark. By [3, Sect.4], for any minimal constant angle surface in $S^{3}(c) \times R$ with $0<|T|<1,|a|$ is positive constant and $b$ is zero. It is not certain if the 
converse is true. So we can consider the following:

Question. Except for minimal constant angle surfaces, does there exist a minimal surface in $N^{3}(c) \times R$ with $c \neq 0$ such that the semi-major axis of the curvature ellipse is positive constant and the semi-minor axis of the curvature ellipse is zero?

Acknowledgements. The author wishes to thank the referee for useful comments.

\section{References}

[1] U. Abresch and H. Rosenberg, A Hopf differential for constant mean curvature surfaces in $S^{2} \times R$ and $H^{2} \times R$, Acta Math. 193 (2004), 141-174.

[2] F. Burstall, D. Ferus, K. Leschke, F. Pedit and U. Pinkall, Conformal geometry of surfaces in $S^{4}$ and quaternions, Lect. Notes in Math. 1772, Springer, Berlin (2002).

[3] D. Chen, G. Chen, H. Chen and F. Dillen, Constant angle surfaces in $S^{3}(1) \times R$, Bull. Belg. Math. Soc. Simon Stevin 19 (2012), 289-304, arXiv:1105.0503vl (math.DG).

[4] Q. Chen and Q. Cui, Isometric immersions into $S^{m} \times R$ and $H^{m} \times R$ with higher codimensions, Results Math. 57 (2010), 319-333.

[5] M. Dajczer and R. Tojeiro, All superconformal surfaces in $R^{4}$ in terms of minimal surfaces, Math. Z. 261 (2009), 869-890.

[6] F. Dillen, J. Fastenakels, J. Van der Veken and L. Vrancken, Constant angle surfaces in $S^{2} \times R$, Monatsh. Math. 152 (2007), 89-96.

[7] D. Fetcu, A classification result for helix surfaces with parallel mean curvature in product spaces, preprint, arXiv:1312.3196vl (math.DG).

[8] D. Fetcu and H. Rosenberg, Surfaces with parallel mean curvature in $S^{3} \times R$ and $H^{3} \times R$, Michigan Math. J. 61 (2012), 715-729.

[9] I. V. Guadalupe and L. Rodriguez, Normal curvature of surfaces in space forms, Pacific J. Math. 106 (1983), 95-103.

[10] D. Hoffman and R. Osserman, The geometry of the generalized Gauss map, Mem. Amer. Math. Soc. 28 (1980), no. 236.

[11] G. D. Johnson, An intrinsic characterization of a class of minimal surfaces in constant curvature manifolds, Pacific J. Math. 149 (1991), 113-125.

[12] G. Ruiz-Hernandez, Minimal helix surfaces in $N^{n} \times R$, Abh. Math. Semin. Univ. Hambg. 81 (2011), 55-67. 
[13] M. Sakaki, Four classes of surfaces with constant mean curvature in $S^{3} \times R$ and $H^{3} \times R$, Results Math. 66 (2014), 343-362.

[14] R. Tribuzy and I. V. Guadalupe, Minimal immersions of surfaces into 4-dimensional space forms, Rend. Sem. Mat. Univ. Padova 73 (1985), 1-13.

Graduate School of Science and Technology

Hirosaki University

Hirosaki 036-8561

Japan

E-mail: sakaki@hirosaki-u.ac.jp 